\title{
La Gouvernance des migrations: de la gestion migratoire à la protection des migrants
}

\section{Alexandra Castro Franco*}

\section{RÉSUMÉ}

Migrants en détresse, tensions aux frontières, crises humanitaires et peurs liées au phénomène migratoire sont devenus un refrain quotidien et médiatique dans toutes les régions du monde. En effet, le phénomène migratoire met à l'épreuve de façon très concrète et immédiate la souveraineté des états, et pose un grand nombre de questions d'ordre juridique, de difficile résolution. Cet article offre une introduction synthétique et pédagogique à la question de la gouvernance des flux migratoires et aux défis que représentent pour les états leur contrôle et la régulation. Par ailleurs, l'auteure décrit les grandes lignes des possibles scénarios qui doivent être explorés pour construire une gouvernance des flux migratoires qui permettent d'articuler les régimes juridiques nationales et internationales pour protéger les états et les migrants, dans le cadre d'un monde globalisé.

Mots-clés: migrations internationales, gouvernance des migraciones, droit des migrants.

\footnotetext{
* Doctora en Derecho Internacional. Docente Investigador, Departamento de Derecho Constitucional, Universidad Externado de Colombia, Bogotá (Colombia). alexandrac.castro@uexternado.edu.co

Este artículo forma parte de la tesis doctoral sustentada por la autora el 12 de mayo de 2014 para obtener el título de doctora en derecho de la Universidad Paris 2 Panthéon Assas. Tesis titulada: La gouvernance des Migrations: de la gestion migratoire à la protection des migrants. Bajo la dirección de M. Emmanuel Decaux.
}

Recibido: 17 de marzo de 2015 / Modificado: 19 de abril de 2015 / Aceptado: 25 de mayo de 2015.

Para citar este artículo

Castro Franco, A. (2015). La Gouvernance des migrations: de la gestion migratoire à la protection des migrants. OASIS, 22, 117-141. DOI: http://dx.doi.org/10.18601/16577558.n22.06 


\section{La gobernanza de la migración: de la gestión migratoria a la protección de migrantes}

\section{RESUMEN}

Migrantes desamparados, tensiones fronterizas, crisis humanitarias y miedos asociados al fenómeno migratorio se han convertido en un refrán cotidiano y mediático en todas partes del mundo. En efecto, los flujos migratorios internacionales ponen a prueba de manera muy concreta e inmediata la soberanía de los Estados, y, en este sentido, plantean un gran número de preguntas de orden jurídico, de muy difícil resolución. Este artículo ofrece una introducción sintética y pedagógica a la cuestión de la gobernanza de los flujos migratorios y a los retos que el necesario control y regulación de estos plantean a los Estados. La autora describe algunos escenarios que deben ser explorados para construir una gobernanza de las migraciones que permita articular de manera efectiva los regímenes jurídicos nacionales e internacionales, para proteger a los Estados y proteger a los migrantes en el marco de un mundo móvil y globalizado.

Palabras clave: migraciones internacionales, gobernanza de las migraciones, derechos de los migrantes.

\section{The governance of migration: from migration management to the protection of migrants}

\section{ABSTRACT}

Migrants in distress, border tensions, humanitarian crises, and fears related to the migration phenomenon have turned into an everyday media issue everywhere in the world. In effect, international migration flows challenge in a very concrete and immediate way the sovereignty of states, and in this sense they pose a large number of very difficult to resolve judicial questions. This article offers a pedagogical and summarizing introduction to the issue of the governance of migration flows and the challenges that the necessary control and regulation of these pose to states. The author describes some scenarios that must be explored in order to construct a governance of migration that will allow the effective articulation of national and international judicial regimes, in order to protect the state and the migrants in the framework of a globalized and mobile world.

Keywords: International migrations, migration governance, migrant rights.

Les migrations existent depuis toujours, elles constituent un phénomène inhérent à l'être humain, une pratique de survie à laquelle l'homme fait appel dans sa recherche permanente de meilleures conditions de vie. C'est ainsi que les mouvements migratoires émer- 
gent comme stratégie pour faire face aux changements climatiques, aux périodes de crises, aux guerres, à d'autres conditions d'adversité ou, simplement, comme un moyen pour chercher de meilleures opportunités dans d'autres endroits du monde.

Les migrations sont une forme de mouvement de population. Au sens large nous entendons par «migration» tout déplacement de personnes. Dans un sens plus strict, nous nous limitons à définir les migrations comme les mouvements de populations qui s'effectuent avec la volonté de demeurer dans l'endroit de destination.

Certains utilisent le terme «mobilité» au lieu du terme «migrations» dans une acception plus large du terme. D'autres les confondent en parlant de mobilité lorsqu'en réalité ils veulent parler de migrations au sens strict. À vrai dire, lorsque nous parlons des migrations, nous retrouvons plusieurs définitions possibles. Cela est dû, en grande partie, au fait que l'étude et la régulation du phénomène migratoire a intéressé pendant très longtemps, de manière exclusive, le droit interne des Etats ${ }^{1}$. Ainsi, chaque Etat a doté d'un sens différent les termes employés. Aujourd'hui la communauté internationale est d'accord sur l'emploi du terme migrations pour décrire les mouvements de population qui se font de manière volontaire et avec une volonté de permanence. Ainsi, les touristes ne sont pas des migrants car ils n’ont pas la volonté de rester dans les pays de visite.
Traditionnellement, les migrations sont comprises comme pouvant regrouper diverses catégories. D'une part, il convient de distinguer entre migrations volontaires, lorsque les migrants ont tous les éléments pour prendre de manière libre et sans contraintes la décision de migrer, inspirés par des raisons d'intérêt personnel. Ceci s'oppose aux migrations involontaires, qui recoupent les situations dans lesquelles les personnes sont obligées de fuir leur foyer du fait de la persécution de tiers (pour des raisons politiques, nationales, d'appartenance à un groupe social, entre autres causes) ou du fait de bouleversements naturels (changements climatiques par exemple). Dans ce cas nous ne parlons pas de migrants mais de déplacés internes (ou victimes du déplacement interne) ou de demandeurs d'asile.

D'autre part, nous distinguons entre migrations internes, lorsqu'elles ont lieu à l'intérieur des frontières de l'Etat et migrations internationales, lorsque les migrants traversent les frontières d'un autre Etat.

Le phénomène migratoire a un impact sur l'économie, la vie sociale, politique et culturelle des Etats tant d'origine que de destination, ainsi que sur la vie et le bien-être des migrants et des membres de leurs familles. Toutefois, pendant très longtemps, il a intéressé exclusivement les pays de destination, qui, à travers des régulations à l'entrée et au séjour des migrants, ont tenté de contrôler leur arrivée. Avec l'avènement de la mondialisation, le phénomène migratoire a acquis une nouvelle dimension

C'est pourquoi, dans le cadre des tentatives pour établir un dialogue mondial sur les migrations, l'orm a élaboré d'un glossaire des termes liés au phénomène afin de les clarifier, d'éviter des ambiguïtés (orm, 2007). 
pour devenir un phénomène mondial qui concerne tous les pays du monde. De nos jours nous considérons que tous les pays sont des points d'origine, de transit et de destination de migrants, c'est pourquoi la seule manière de maîtriser les migrations passe par les efforts conjoints de toutes les parties concernées. C'est ici qu'apparaît le besoin d'une gouvernance des migrations ${ }^{2}$, entendue comme un effort conjoint de la communauté international dans son ensemble afin de parvenir à la maîtrise des migrations à travers la coopération, l'échange et l'entraide.

Cette gouvernance s'est avérée compliquée du fait des intérêts contradictoires des Etats, mais aussi en raison du manque de prise de conscience de ces derniers sur les enjeux des migrations, ses causes et conséquences et même de leur histoire. C'est pourquoi, cet article vise à réviser l'histoire, les causes et conséquences des migrations avant de montrer comment la gouvernance des migrations présente un défi de taille pour les Etats dans ses relations avec ses proches spécialement pour ce qui touche à l'accomplissement des obligations internationales des Etats en matière de protection des droits de l'homme.

\section{L'HISTOIRE DES MIGRATIONS}

L'histoire des migrations est aussi ancienne que celle de l'humanité. Nous rencontrons des traces très anciennes de mesures prises pour limiter les droits des étrangers (mot qui vient du latin extranêus et signifie celui de dehors, qui n'appartient pas à la famille). En Egypte ancienne par exemple, les étrangers étaient exclus des cercles sociaux et les travaux les plus dégradants leur étaient confiés. Ils risquaient même d'être sacrifiés aux dieux. Dans les pyramides on trouve l'inscription «aucun homme du pays n'y a travaillé» (Villegas, 1919, p. 4). En Grèce antique, les citoyens des autres villes-Etats étaient dépourvus de droits et toute relation de type commerciale avec eux était proscrite (De Soigne, 1873). Dans la Rome antique, ils sont passé du statut d'exclus leur refusant tout droit, à un statut plus favorable, obtenu en raison des besoins commerciaux ${ }^{3}$. Dans la Bible, à l'inverse, tant dans l'Ancien que dans le Nouveau Testament, on retrouve des références précises sur le respect et la protection des migrants indépendamment de leur statut juridique (Cervantes Gabarrón, 2008). Les migrations ont été présentes à tous les moments de l'histoire c'est pourquoi nous

\footnotetext{
2 Il convient de préciser que le terme "gestion» très utilisé pour parler de la maîtrise des migrations suscite beaucoup de commentaires. Il a été critiqué par ceux qui trouvent que le terme est inapproprié car il déshumanise le phénomène migratoire. Le terme «gouvernance des migrations» paraît plus approprié et plus humain c'est pourquoi son utilisation a été conseillé FMMD sixième réunion du Forum mondial sur migration et développement, Port Louis, Mauritius 1922 Novembre 2012.

3 Les institutions de l'hospitium et du patronat sont cités dans ce sens. Madiedo. Droit des gens 1863 p 200 numéro 100. cité dans Op cit p. 5.
} 
pouvons dire, sans nous tromper, que toutes les régions du monde ont été touchés par le phénomène. Pensons à l'époque du mouvement de découvertes accompagnée de la traite d'esclaves en provenance d'Afrique et des déplacements internes au sein du continent américain.

Aux temps modernes, on trouve des références au droit appartenant aux Etats de fermer leurs frontières dans des pratiques qui datent du début du 19 ème siècle ${ }^{4}$, ainsi que d'autres règles à caractère coutumier motivées par la présence d'occidentaux dans les pays du sud. Ces mesures n'obligeaient pas les Etats à admettre les étrangers mais prévoyaient qu'une fois sur le territoire, un minimum de droits leur seraient garantis. A l'époque, la protection diplomatique et consulaire est apparue comme un moyen de garantir l'action de l'Etat d'origine des migrants en cas de violation de leurs droits.

Par ailleurs, la fin de la seconde guerre mondiale a marqué le début d'une nouvelle tendance migratoire. Le développement économique des Etats Unis et d'autres Etats européens s'est accompagné d'une demande de main d'œuvre dans les secteurs de la construction et de la manufacture. C'est ainsi que des travailleurs en provenance de pays périphériques tels que l'Italie, l'Espagne et la Grèce, entre autres, ont migré pour répondre à ces demandes grâce à des systèmes comme celui des «travailleurs invités» (Castles y Miller, 2004 cité dans Martinez P., 2008, p 34). Les premières règles sur les droits des travailleurs migrants de l'oIT $^{5}$ datent également de cette époque. L'Amérique du Sud a également connu de grandes vagues de migrations en provenance d'Europe au cours de cette période.

Plus tard, le panorama migratoire a de nouveau changé. Les crises économiques et les disparités croissantes entre pays développés et pays en voie de développement ont marqué une nouvelle tendance vers des restrictions migratoires, notamment à partir des années soixante-dix, restrictions qui n'ont cessé de se multiplier depuis, notamment avec l'avènement de la mondialisation, accompagnée de la massification des migrations.

En effet, avec la mondialisation, les migrations ont gagné une telle ampleur qu'elles se sont placées au cœur des inquiétudes des sociétés. Pour certains, comme Saïd (2005, p. 17), elles ont été l'événement le "plus important des trois derniers décennies ». Elles sont cataloguées comme le fruit des tendances modernes, mais en même temps elles constituent un fort propulseur de nouvelles dynamiques propres au contexte de la mondialisation. Cette double valeur des migrations implique bien entendu des confrontations entre des intérêts parfois contradictoires. La mondialisation est un processus qui implique la multiplication des échanges et de la circulation au niveau social, économique et humain. Ce phénomène est encouragé par la mise en valeur, la multiplication et la massification des moyens de communication et de transport.

\footnotetext{
4 Comme il a été fait au Paraguay par le dictateur Gaspar entre 1814 et 1838.

5 Convention 97 de l'orT révisée en 1949.
} 
D'un côté nous pouvons dire que l'accroissement des migrations a servi de catalyseur de la mondialisation car les migrations ont attiré de la main d'œuvre bon marché et spécialisée pour assurer une production à échelle globale. D'un autre côté, les migrations sont le produit de la mondialisation car elles sont le fruit des tendances d'échange, de massification et d'ouverture que nous venons de décrire.

Par ailleurs, l'impact de la mondialisation entraîne des changements dans le cœur même de la société et des structures traditionnelles du pouvoir, ce qui implique des nouvelles perceptions du territoire ainsi de nouveaux rapports économiques et sociaux ${ }^{6}$. La conception de l'Etat et de ses frontières est également impactée par la mondialisation. Le contrôle des frontières et l'homogénéité des sociétés sont questionnées face à une réalité qui fait changer la nature traditionnelle de l'Etat-Nation (Sassen, 2001, p. 49).

Les Etats s'adaptent certes plus ou moins facilement aux effets économiques de la mondialisation. Ils sont conscients des changements qui s'opèrent dans les relations d'échange économique, ainsi que concernant l'ouverture des marchés et la libre circulation des biens. Cependant, ils ne sont pas prêts à abandonner leur conception traditionnelle de nation et continuent à «revendiquer leur ancienne splendeur en affirmant leur droit souverain à contrôler leurs frontières» (Sassen, 2001, p. 73). La mobilité des personnes est encore perçue comme un outrage aux principes nationalistes exclusifs que conçoivent l'Etat national comme celui qui s'identifie uniquement avec un territoire. De ce fait, tous ceux qui n'appartiennent pas à un Etat et qui ne partagent pas les valeurs nationales sont perçus comme une menace contre la stabilité et de ce fait doivent être maîtrisés.

Les Etats ont du mal à comprendre que les attributs de la souveraineté ne disparaissent pas avec la mondialisation, bien qu'elle comporte indéfectiblement des nouvelles nuances dans son exercice. Le monde n'est plus celui des sociétés homogènes et isolées, les frontières sont chaque fois plus poreuses, c'est à dire, plus faciles à traverser, et la protection des droits de l'homme s'impose, au moins en théorie, même à l'encontre de la volonté des états.

Ces nuances comportent des tensions, notamment en ce qui concerne la protection des droits des migrants en situation irrégulière qui, ayant traversé les frontières ou étant restés à l'intérieur sans autorisation, continuent néanmoins à être titulaires de droits ${ }^{7}$. C'est pourquoi nous pouvons affirmer, avec Sassen, que

Les immigrants et les réfugiés montrent la tension existante entre la protection des droits de l'homme et la souveraineté des Etats. Cette tension

6 Dans les termes de Sassen il s'agit d'un changement dans la «géographie du pouvoir» (Sassen, 2001, p. 25).

7 A ce stade nous souhaitons préciser ce que nous comprenons par migrant en situation irrégulière: il s'agit du migrant qui accède sans autorisation au territoire d'un Etat (qui n'est pas son Etat d'origine); qui y accède frauduleusement ou qui a décidé d'y rester une fois son autorisation de séjour expirée. Certains utilisent le terme migrants «clandestins ». Nous considérons qu'aucune personne ne peut pas être clandestine c'est pourquoi nous n'utiliserons pas ce terme. 
s'avère particulièrement aigüe s'agissant des migrants en situation irrégulière car leur simple existence implique une érosion de la souveraineté (Sassen, 2001, p. 73).

En effet, bien que la circulation des personnes, leur entrée et sortie d'un territoire déterminé corresponde à l'exercice d'une prérogative légitime des Etats, dans la jouissance de leur souveraineté, les transformations qu'a subi ce dernier concept par le biais de la modernisation et l'édification de l'individu comme titulaire de droits à vocation universelle ont compliqué le contrôle des frontières qui, bien qu'il continue à être totalement en vigueur, comporte des difficultés. Aujourd'hui nous comprenons que la souveraineté implique ainsi une double responsabilité. Tel que cela a été souligné par la Commission internationale sur l'intervention et la souveraineté des Etats en 2001, elle englobe la responsabilité «externe - respecter la souveraineté des autres États - et interne - respecter la dignité et les droits fondamentaux de toute personne vivant sur le territoire de l'État" (CIISE, 2001, p. 9). Cette souveraineté ne se traduit par un pouvoir d'action illimité des Etats, et «tout comme les individus ont des droits et des responsabilités en tant que citoyens d'un Etat, les Etats ont des droits et des responsabilités en tant que membres de la communauté internationale» (Commission Mondiale sur les migrations internationales, 2005). De cette manière, bien que les Etats sont libres de déterminer qui entre et qui sort de leur territoire, une fois ces personnes arrivées, un minimum de droits doit leur être garanti.

Nous avons expliqué comment à travers l'histoire, les migrants ont fait l'objet de préjugés, restrictions et contrôles. Cette tendance n'a pas faibli avec l'avènement de la mondialisation. Ainsi, une conception négative des migrants consistant à dire qu'ils seraient nocifs pour les sociétés et à les accuser de tous les maux économiques, financiers ou sociaux, comme par exemple de la délinquance ou la de la propagation de maladies, continue à être courante dans certains endroits du monde. La situation de tension et de malaise autour des migrants, est due, en partie, comme l'expliquent plusieurs auteurs tels que Kymlicka (2002, p. 267), à la peur générée par la diversité au sein de la société. Sans vouloir entrer dans des débats d'ordre sociologique, nous nous contenterons de dire que cette crainte a irradié toutes les sphères de la société, dans un processus de "construction sociale d'un ennemi» ${ }^{8}$ et teinte les tentatives de gestion migratoire d'une nuance sécuritaire. Cette situation complique davantage la tâche des Etats devant exercer leur souveraineté pour éviter l'arrivée des migrants «indésirables» tout en accomplissant leur obligation de respecter et de protéger les droits humains de ceux qui se trouvent déjà sur le territoire (Sassen, 2001).

L'analyse de l'histoire des migrations montre comment il s'agit d'un phénomène existant depuis l'ancien temps, auquel l'homme a toujours fait appel en temps de crise comme une stratégie de survie ou toute simplement

8 Commission des droits de l'homme. Résolution 1999/80 du 9 mars 1999. 
comme un moyen pour satisfaire sa curiosité. Etat un phénomène connaturel à l'être humain c'est évident que toute tentative cherchant à éliminer les migrations est inapplicable car les personnes continueront à trouver des moyens pour migrer. Analysons à présent les causes et conséquences des migrations.

\section{CAUSES ET CONSÉQUENCES DES MIGRATIONS}

L'intérêt suscité par les migrations a entrainé l'émergence de tout un ensemble de théories cherchant à expliquer les causes du phénomène. Sans entrer dans l'étude des multiples théories existantes, telles que la théorie fonctionnaliste ${ }^{9}$ et historico-culturelle propres à la sociologie et à l'économie, nous nous bornerons à mentionner les principales théories qui ont été abordées par les chercheurs et qui ont inspiré la prise de mesures pour maitriser les migrations. En premier lieu, la théorie du "push-pull" (expulsion et attraction), selon laquelle les flux de travailleurs correspondent d'une part aux conditions de vie difficiles adverses des pays d'origine qui poussent les migrants à sortir, et d'autre part aux facteurs d'attraction des pays de destination qui invitent les migrants à tenter leur chances à la recherche de meilleurs salaires, de stabilité politique ou d'une structure sociale dans ces pays. La combinaison de ces deux facteurs inciterait les migrants en provenance des couches les moins favorisés et des régions les plus reculées du monde, à se déplacer vers les pays les plus développés. Cette théorie a inspiré la plupart des mesures adoptées par les pays de destination en vue de stopper l'arrivée des migrants par le biais d'aides au développement dans les pays d'origine des migrants. Cependant, selon d'autres études, les migrations ne sauraient simplement s'expliquer par le jeu de l'offre et la demande. Bien que ces facteurs aient du poids, le parcours migratoire se construit autour d'autres relations plus profondes et un ensemble plus complexe de motivations qui dépassent le cadre purement économique. Par ailleurs, si la théorie du "pull and push» pourrait expliquer le départ de migrants non qualifiés qui fuient la pauvreté elle n'explique pas celle des professionnels, scientifiques et hommes d'affaires. De la même manière, cette théorie s'est avérée insuffisante pour expliquer pourquoi ces forces d'attraction et d'expulsion ne poussent pas toute la population des pays en voie de développement à partir, attirés par les opportunités des pays développés ${ }^{10}$.

Deuxièmement, est ensuite apparue une théorie qui se détache des analyses purement économiques des migrations pour ajouter des éléments sociologiques dans l'étude des mouvements migratoires, de ses causes et de ses conséquences. Par le biais de travaux d'observation, cette théorie a conclu à une alternative qui semble plus satisfaisante pour

\footnotetext{
9 A ce sujet voir (Guarnizo, 2006, p. 70).

10 Des théories postérieures telles que la théorie marxiste ou la théorie dit du «système mondial» ont aussi expliqué les migrations comme correspondant à des motivations purement économiques mais dictées, non pas par une analyse de la part des acteurs mais plutôt comme une conséquence du système capitaliste.
} 
expliquer le phénomène migratoire et qui invite à adopter une perspective transnationale des migrations (Guarnizo, 2006, p. 80). Cette façon d'interpréter les migrations, qui a surgi dans les années quatre-vingt mais a regagné du terrain à partir du $21^{\text {ème }}$ siècle, comprend les migrations comme un processus

...dynamique de construction et reconstruction des réseaux sociaux qui structurent la mobilité spatiale et la vie du travail, sociale, culturelle et politique tant de la population migrante que celle de leurs familles, amis et les communautés dans les pays d'origine et de destination (Guarnizo, 2006, p. 80).

D'après cette théorie, les migrants ne sont pas isolés dans leur décision de migrer, ni dans leur choix du pays de destination et du parcours migratoire; ils ne sont pas guidés exclusivement par des motivations personnelles et indépendantes. Les migrations sont la conséquence de tout un processus de réflexion conditionné par l'entourage social, économique et politique des migrants. Ainsi, la famille, les amis et même les membres d'un groupe social, tant dans les pays d'origine que de destination, sont déterminants pour les migrants dans la construction d'un projet de vie et de la prise de décision de migrer. Ils continuent également à l'être dans la vie quotidienne dans les pays de destination à travers les forts liens que les migrants gardent et nourrissent avec leurs sociétés d'origine. Ainsi, nous ne pouvons plus parler d'une séparation radicale entre pays d'origine et de destination des migrants car de forts liens sont cultivés entres les deux, dans un phénomène transnational ${ }^{11}$. Les migrants ne partent pas vers les pays de destination les mains vides, ils emportent avec eux leur culture et leurs coutumes; tout en essayant de s'adapter à la société de destination ils gardent des engagements, des relations et de fortes attaches avec leurs sociétés d'origine. Ces relations peuvent être d'ordre familial, avec de forts liens de dépendance économique et affective, entretenus de nos jours grâce aux progrès technologiques qui ont facilité les communications et les échanges virtuels. Le degré d'intensité des liens est influencé par les caractéristiques des pays d'origine et le contexte particulier (rural ou urbain) d'où provient le migrant. Ainsi les migrants en provenance de zones rurales seraient plus enclins à garder des liens solides avec leurs proches et à développer une solidarité forte avec les migrants en provenance de la même région géographique dans les pays de destination. S’agissant des colombiens, par exemple, de forts liens entre les migrants provenant du village de Santuario (un petit village dans le département d'Antioquia) se sont développés à Paris ${ }^{12}$, ce qui contraste avec la faible tendance au rapprochement entre migrants provenant des grandes villes.

Pour certains le «transnationalisme», loin d'être une tendance voulue, est plutôt une «stratégie obligatoire de survie des migrants» (Martínez Pizarro, 2010, p. 74), conséquence

\footnotetext{
11 Nous parlons d'un phénomène transnational car il dépasse les frontières d'un seul Etat pour établir des relations simultanées entre deux ou plus Etats.

12 A ce sujet voir les travaux de Gincel (2007, pp. 32-42).
} 
des conditions difficiles auxquelles ils doivent faire face dans les pays de destination. De notre point de vue, cette théorie explique bien les dynamiques des migrations latino-américaines, très influencées par les réseaux familiaux qui s'étendent sur plusieurs générations et déterminent le comportement de ses membres, et leur façon d'agir, même lorsqu'ils se trouvent à l'étranger ${ }^{13}$.

Au-delà de la théorie à laquelle on adhère pour expliquer les flux migratoires, un constat s'impose dans l'étude des migrations: il s'agit d'un phénomène inhérent à l'être humain qui existe et se perpétuera. C'est pourquoi les idées d'une société fermée ou d'une migration totalement contrôlée sont irréalistes ou inapplicables.

Ayant analysé les causes des migrations, passons à l'étude de ses conséquences, réelles et fictives, également utilisées comme arguments pour justifier les mesures de gestion migratoire adoptées par les pays de destination des migrants.

S'agissant des conséquences des migrations, compte tenu du caractère transnational des migrations, il va de soit que les migrations produisent des effets tant dans les pays d'origine que de destination. Ces conséquences, bien entendu, sont plus fortes dans des contextes de migration massive et dans de tels cas, les mesures prises pour $y$ faire face sont plus radicales. En outre, si ces effets peuvent être réels, ils s'avèrent parfois exagérés par les perceptions sociales. Commençons par évoquer l'impact, réel ou ressenti, des migrations sur les pays de destination.

En premier lieu, la migration massive des personnes implique nécessairement des changements dans les structures sociales et culturelles des sociétés de destination. Ce mélange de cultures et de traditions est un résultat indéniable de la mondialisation qui touche aux idées d'identité nationale, d'appartenance à une nation et ses mœurs.

Les sociétés de destination traditionnellement attachées à des principes, valeurs et coutumes, à une histoire et à des traditions communes, voient leur paysage changer du fait de l'arrivée d'étrangers. La conception d'un Etat Nation homogène est ainsi mise en doute par la cohabitation de plusieurs cultures et de plusieurs communautés ayant des conceptions et des pratiques sociales et culturelles diverses. Pour certains il s'agit d'un effet nocif des migrations qui menacent les traditions et culture locales; pour d'autres, au contraire, c'est un moyen d'enrichir ces cultures et leur donner une nouvelle dimension post moderne.

13 D'après les études de l'orm d'autres courants se concentrent sur l'analyse des facteurs qui déterminent les migrations pour distinguer entre facteurs habilitants qui favorisent et rendent possible le départ des migrants tels que les changements géopolitiques, l'évolution des médias et des moyens de transport et la création des réseaux transnationaux. (Dans cette perception, la dimension transnationale des migrations ne servirait pas de cause aux migrations mais plutôt d'élément facilitateur). Et des facteurs causatifs comme étant les responsables directs des migrations, lesquels se dérivent de la conjugaison de plusieurs «variables socio-économiques» tels que le marché du travail et ses taux de rémunération, les facteurs démographiques, l'accès à certains biens et services, la qualité de vie, le régime politique en place et même les facteurs climatiques (orm, 2010, p. 2). 
Deuxièmement, les migrations impactent le marché du travail des sociétés de destination. Pour certains, les migrants sont une menace pour ces marchés car ils viennent occuper une place qui devrait être destinée aux nationaux. Il est indéniable que les migrants impactent les marchés de travail. Toutefois, plusieurs études ont aidé à démontrer que la plupart des migrants ne concourent pas pour les emplois des natifs, mais pour exercer des activités que les nationaux ne veulent ou ne peuvent pas exercer, cela se produit même en dépit du niveau de qualification parfois élevé des migrants, qui pourrait souvent leur permettre d'opter pour un parcours différent. Dans ce sens, le Programme des Nations unies pour le développement (PNUD) (2009) ainsi que la Commission mondiale sur les migrations internationales ont reconnu que les migrants occupaient des travaux non attractifs pour les nationaux et des activités qui nécessitent une haute qualification que les locaux ne possèdent parfois pas. Par ailleurs, le Rapport de ladite Commission indique que «Dans certains pays, des secteurs entiers de l'économie et de nombreux services publics sont devenus fortement dépendants du travail migrant et s'effondreraient très rapidement si ces travailleurs n'étaient plus disponibles» (Commission Mondiale sur les migrations internationales, 2005, p. 5). Toujours dans le domaine du travail, certains considèrent que les migrants font baisser la moyenne des salaires car ils sont prêts à accepter une rémunération plus faible en échange de leur travail. Cette situation est récurrente mais s'explique en grande partie par les difficultés que rencontrent les migrants pour accéder au marché de travail des pays de destination, ce qui les oblige à accepter des rémunérations plus faibles.

Troisièmement, les migrations impliquent un coût financier pour les pays de destination $\mathrm{du}$ fait de l'accès des migrants aux services sociaux tels que l'éducation et la santé. Certains trouvent que ce coût est excessif et que, de ce fait, les migrations devraient être contrôlées ou l'accès des migrants à ces services restreint. D’autres ont estimé que de la même façon que les migrants utilisent les services sociaux des Etats, ils doivent contribuer à leur financement. Pour certains pays du monde, cette contribution est si importante qu'elle est considérée comme étant nécessaire pour garantir la viabilité desdits services notamment dans des contextes de vieillissement démographique où l'apport des migrants s'avère capital pour garantir le financement des systèmes de retraite ${ }^{14}$.

Enfin, les théories sur les prétendus liens entre migrations et menace sécuritaire existent depuis bien longtemps. Les migrants sont les boucs émissaires pour expliquer les problèmes sécuritaires, les dangers publics et même les problèmes sanitaires du fait de leur caractère prétendument dangereux et suspect. Les attentats du 11 septembre 2001 ont contribué à renforcer les conceptions présentant les migrants comme un danger pour la société et une menace contre la sécurité, comme cela fut rappelé lors de l'Initiative de Berne (Office fédéral des migrations, oDM, OIM, 2004) et dans

14 Concernant cet aspect voir les études présentés par le PNUD (2009). 
le Rapport de la Commission mondiale pour les migrations internationales (Commission Mondiale sur les migrations internationales, 2005). La migration irrégulière, stratégie de survie pour beaucoup de personnes est considérée comme une menace contre la sécurité et la sûreté des Etats, et qualifiée de délit dans plusieurs législations pénales. Certains chercheurs se sont même employés à prouver la «dangerosité» des migrants par le biais de statistiques biaisées du nombre de crimes commis par les personnes d'ascendance étrangère ${ }^{15}$.

En somme, le phénomène des migrations n'a rien d'anodin pour les sociétés de destination. Leurs implications sont non seulement d'ordre culturel et social mais aussi démographique; elles impactent le marché du travail, les services sociaux ainsi que le commerce, le tourisme, le transport aérien et les habitudes de consommation.

Comme nous l'avons déjà dit, il est difficile d'établir quelles sont les véritables conséquences des migrations tant dans les pays d'origine que de destination. En effet, aux effets réels du phénomène s’ajoutent les conséquences problématiques dont l'impact est exagéré par la société. Les attitudes négatives vis-à-vis des migrants sont persistantes, et sont parfois entretenues par les médias. Elles nourrissent les discours électoraux des partis d'extrême droite, lesquels se traduisent par des pratiques et comportements racistes et xénophobes à l'encontre des migrants ${ }^{16}$.

Il convient de souligner que les décisions concernant l'entrée et le séjour des migrants font partie de l'exercice légitime d'une prérogative propre de la souveraineté des Etats de destination (Assemblée Générale, 2002). Dans ce sens, les Etats conditionnent l'accès et le séjour des étrangers sur leur territoire en fonction de leurs besoins ponctuels et du contexte social et économique. Ces mesures ne sont pas mauvaises en soi mais peuvent devenir un instrument véhiculant les craintes parfois injustifiées à l'encontre des étrangers et de ce fait, constituer un moyen de discrimination infondé envers ses personnes et favoriser leur isolement.

Après avoir abordé les conséquences des migrations pour les pays de destination nous allons répéter l'exercice pour les pays d'origine des migrants. Ces pays subissent des effets démographiques, économiques et sociaux du fait du départ d'une partie de leur population, conséquences qui ont un impact renforcé lorsque la sortie des nationaux est massive.

Tout d'abord, le départ des nationaux d'un pays implique l'allègement d'une partie de la charge sociale de l'Etat. Ces nationaux qui demandent emplois, éducation et services sociaux sortent du pays et diminuent de ce fait les devoirs de l'Etat à leur égard. La contrepartie de cet effet qui pourrait être regardé comme

\footnotetext{
15 Notamment Gourévitch (2009) dans laquelle l'auteur prétend montrer que les migrants coûtent plus que ce qu'ils ne rapportent en utilisant des statistiques biaisées.

16 Ce type de tendances et pratiques a été fortement condamné par le Rapporteur spécial des Nations unies sur les droits de l'homme des migrants, le Rapporteur spécial sur les formes contemporaines de racisme et le Rapporteur sur les droits de tous les travailleurs migrants. Voir entre autres, Commission des droits de l'homme (1999); Assemblée générale des Nations Unies (2013).
} 
positif, est la perte de capital humain, la fuite de cerveaux ou brain-drain ${ }^{17}$. Les Etats investissent dans l'éducation de leurs nationaux qui constituent la force de travail de l'Etat, contribuent au financement du système et sont le moteur de développement du pays. Or il s'avère que les migrations attirent particulièrement les secteurs les plus éduqués de la population. Ainsi, les Etats d'origine voient partir leurs médecins, leurs ingénieurs et d'autres travailleurs hautement qualifiés, qui auraient pu apporter au développement du pays, créer des emplois et aider dans la formation d'autres personnes. Vu sous un angle positif, la fuite de cerveaux peut encourager le transfert de technologie et du savoir faire depuis les pays développés vers les économies émergentes. Dans une telle optique, les personnes qualifiées qui sont parties peuvent garder et entretenir des liens avec le pays d'origine, ce qui peut se traduire en un échange de connaissances. Toutefois, la réalité montre trois situations qui rendent cet échange hypothétique complexe. En premier lieu, bien qu'hautement qualifiées, ces personnes sont bien souvent employées pour des travaux demandant peu de qualification, ce qui constitue un "gaspillage» de leurs capacités ${ }^{18}$. Par ailleurs, ces personnes se limitent souvent à n'entretenir que des liens strictement familiaux avec leur pays d'origine. Si elles sont d'abord parties en raison du manque d'opportunités professionnelles dans leurs pays, c'est aussi du fait du désintérêt de leurs gouvernements pour encourager la recherche et les projets d'innovation. De ce fait, elles sont rarement satisfaites de la gestion de leurs Etats et n'ont pas d'intérêt à s'investir dans le développement du pays qui leur a tourné le $\operatorname{dos}^{19}$. Finalement, les bénéfices de ce transfert ne compensent pas la perte subie par les pays d'origine qui ont investi dans la formation de ces personnes.

Les Etats d'origine ne sauraient empêcher le départ de leurs ressortissants. Un défi se présente toutefois à ces pays: créer des mesures suffisamment attractives pour tisser des liens avec ces migrants et encourager leur engagement dans des projets bénéfiques pour le pays. De cette manière, la perte de capital humain (brain-drain) peut se transformer en un gain pour le pays d'origine (brain-gain) ${ }^{20}$.

\footnotetext{
17 La Commission mondiale sur les migrations internationales trouve exagéré le terme «exode de cerveaux» car elle suppose que ces personnes qualifiées qui quittent le pays n'y retourneront plus, et considère plus adéquat le terme «circulation des cerveaux ». Nous considérons pour notre part qu'étant donné que le retour des migrants qualifiés qui travaillent à l'étranger n'a en général pas lieu pour travailler dans le pays d'origine mais plutôt pour entretenir les liens familiaux, le terme demeure approprié (Commission Mondiale sur les migrations internationales, 2005, p. 34).

18 Cette situation est très courante s'agissant des migrations en provenance de pays andins et d'Amérique centrale vers les Etats Unis (Mattoo, Aaditya, Ileana et Özden, 2005).

19 Dans ce sens, il est considéré que le succès des politiques de rapprochement des nationaux de l'étranger dépend en grande partie de la confiance que ces personnes ont dans l'État qui les invite à se rapprocher des autorités publiques. Dans ce sens: Rannveig Agunias (2009).

20 C'est notamment le cas de l'Inde, de la Chine et de Taiwan où la croissance économique a motivé le retour massif de nationaux qualifiés qui avaient quitté leur pays Pellegrino (2004).
} 
Qui plus est, une fois dans les pays de destination, les migrants envoient des fonds à leurs familles restées dans les pays d'origine. Ces sommes d'argent ont créé un marché fleurissant et constituent une importante source de revenus pour les Etats d'origine. En effet, le marché du transfert des fonds s'est fortement développé au cours des dernières années. $\mathrm{Ce}$ marché, qui a émergé à l'écart des structures officielles, a fait l'objet d'un manque de régulation dont ont bénéficié les entreprises privées. Ainsi, les intermédiaires financiers, notamment les maisons de change et les banques, ont profité de ce contexte pour pratiquer des transferts à des commissions très élevées (la moyenne de nos jours est de $6 \%$ du montant transféré).

L'Amérique latine dans son ensemble est connue pour étant le réceptacle d'importantes sommes d'argent de la part de ses ressortissants à l'étranger, à tel point que d'après les estimations officielles de l'oim pour l'année 2008, la région reçoit plus de $40 \%$ du volume mondial de versements de fonds, ce qui, par ailleurs «dépasse le montant de l'aide externe que reçoit la région et commence à dépasser les investissements directs étrangers que reçoivent les pays concernés» (OIM, 2002, p. 12) et constitue un montant plus important que celui de l'aide publique au développement. Ces montants ont été moins importants en 2009 en raison de la crise économique mondiale mais demeurent assez élevés ${ }^{21}$. Les pays de la région qui perçoivent la portion la plus élevée des fonds sont la Colombie, le Brésil et le Mexique, dont les envois correspondent à 60\% de l'argent total transféré par les migrants (Martínez Pizarro, 2003, p 83). Dans la région andine, l'Equateur a été le premier pays à réguler le coût du transfert de fonds pour limiter les abus des intermédiaires privés et établir des mécanismes officiels pour l'envoi de fonds.

L'envoi de fonds constitue l'aspect le plus «séduisant» des migrations. Il a motivé une grande quantité d'études, de recherches, de sommets et de bilans visant à ce que ces sommes profitent au développement des pays d'origine des migrants. En effet, dans certains pays, des alliances ont permis de profiter de cet argent pour encourager le développement ${ }^{22}$ et ont servi de moyens d'investissement productif. Toutefois, cela n'est pas toujours le cas. Les fonds envoyés par les migrants, bien que constituant d'importantes sommes d'argent, ne constituent pas nécessairement une source de développement pour les pays d'origine. Bien au contraire, ces fonds sont investis dans la consommation familiale et les dépenses courantes des proches des migrants. C'est-à-dire qu'ils ne sont pas des sources de richesse mais

\footnotetext{
21 Dans ce sens voir: BID (2010).

22 Comme c'est le cas du Mexique où l'argent obtenu par le biais de transferts correspond à quatre fois leur exploitation agricole et deux tiers de leur exportation pétrolière (Martínez Pizarro, 2003). Par ailleurs, d'après la CMMI le cas des Philippines est également un bon exemple pour démontrer que l'envoi de fonds contribue à encourager le développement dans les pays d'origine et peut constituer un outil de lutte contre la pauvreté. Ce même cas a été présenté dans le cadre du Forum mondial sur les migrations et le développement.
} 
des sources de dépendance ${ }^{23}$. Ils permettent de financer les loyers, les fournitures scolaires et les vacances des enfants, des parents et d'autres membres de la famille des migrants. Il ne sert pas à financer des projets productifs. En outre, les familles des migrants paient au prix fort la séparation avec leurs êtres chers, prix qui n'est pas compensé par l'argent envoyé. A cet égard, le Sommet ibéro-américain des migrations n'a pas manqué de signaler que «L'envoi de fonds des migrants n'est ni une panacée pour le développement ni un mécanisme capable de substituer la responsabilité centrale de chaque Etat en matière de politiques de développement et d'équité sociale» ${ }^{24}$. De même, le document signale les avantages que peuvent comporter certains systèmes d'envoi de fonds (notamment ceux des donations des communautés d'immigrés) pour le financement de projets de développement. Le rapport de la Commission mondiale sur les migrations internationales à quant à lui signalé les bienfaits et les risques de l'envoi des fonds tout en invitant les Etats à «réduire les coûts de transfert, surveiller les entreprises qui travaillent sur ce marché ainsi que respecter le caractère privé de ces fonds $»^{25}$.
De manière générale, nous verrons apparaître ce sujet dans le cadre de toutes les discussions sur les migrations et le développement, ainsi que dans les politiques publiques des principaux pays d'origine des migrants. Ces sommes d'argent constituent une sorte de caléidoscope du phénomène migratoire: fruit de l'effort et de lourdes journées de travail des migrants à l'étranger, les sommes envoyées tentent, sans succès, de combler le vide laissé par l'absence des êtres chers. Elles deviennent de l'argent de poche pour certains; un indicateur social pour d'autres; ce qui justifie la permanence à l'étranger de beaucoup; une source de richesse pour les intermédiaires et une fausse source de développement pour ceux qui élaborent les indicateurs économiques, à vrai dire l'envoi de fonds n'a qu'un seul mérite absolument incontestable: celui d'avoir réussi à attirer l'attention des états d'origine sur le sort de leurs ressortissants à l'étranger, et d'avoir contribué à la création d'espaces de discussion à l'échelle mondiale sur les migrations. Bien entendu, cet intérêt fut au départ purement économique mais a commencé à évoluer pour s'élargir à d'autres aspects du phénomène ${ }^{26}$.

\footnotetext{
23 Cette situation a été soulignée à plusieurs reprises par la société civile dans le cadre du Forum mondial sur les migrations et le développement, notamment lors du premier Forum (FMMD, 2008).

24 Xvi Sommet Ibéro-Américain .Montevideo Uruguay, 3, 4 et 5 novembre 2006.

25 Commission Mondiale sur les migrations internationales (2005, p. 30). Dans le même sens voir Assemblée générale des Nations unies (2005). Ce dernier aspect a également été abordé lors de la Conférence internationale de financement pour le développement durable à Monterrey, Mexique, le 18 et 22 mars 2008.

26 L'envoi des fonds n'est pas le seul marché qui s’est développé autour des migrants et leurs familles. Les fortes sentiments qui lient les migrants avec leur communauté d'origine ont encourage le développement de ce qui a été appelé le «business de la nostalgie». Construit autour du besoin des migrants de consommer des produits typiques de leur pays d'origine pour se sentir plus proche de leur foyer, et de voyager avec régularité pour visiter ses proches ce marché propose l'exportation de produits de consommation qui sont vendus dans des locaux et des restaurants spéciaux où la diaspora se réuni pour partager des souvenirs, manger des plats typiques et danser.
} 
Pour compléter notre analyse sur les conséquences des migrations, il est nécessaire d'aborder leur impact sur les migrants et les membres de leurs familles. Tout d'abord il convient de rappeler que le migrant est une personne titulaire de droits et d'obligations à caractère universel. Ainsi, il est non seulement soumis à la juridiction des pays de destination et doit à ce titre respecter les normes d'entrée et séjour qui y ont été établies, mais il doit également pouvoir jouir d'une protection adéquate de ses droits humains, en particulier dans la mesure où la plupart des pays de destination sont signataires des principaux instruments de protection des droits de l'homme ${ }^{27}$. En pratique, ces Etats éprouvent des difficultés à concilier le droit des étrangers, droit «régalien» (Masson, 2010) issu de leur souveraineté, pour contrôler leurs frontières et les personnes qui y circulent, avec les droits de l'homme. Cette réalité se complique davantage s'agissant des migrants en situation irrégulière.
Le migrant est considéré comme étant en situation de vulnérabilité car il se trouve loin de son pays d'origine, de sa culture, de sa famille et de la protection de ses autorités nationales. Cette condition a été reconnue à maintes reprises $^{28}$ et a fait l'objet de plusieurs études qui ont abouti à la conclusion que les migrants

En tant que sujets des droits de l'homme, sont vulnérables parce qu'ils sont privés de reconnaissance et de pouvoir. Ils sont vulnérables parce qu'ils sont considérés comme «extérieurs» aux sociétés d'accueil et ils sont vulnérables parce que les États d'accueil n'appliquent pas les normes internationales relatives aux droits de l'homme ni aux migrants réguliers ni aux migrants irréguliers (Commission des droits de l'homme, 1999).

Cette vulnérabilité des migrants se manifeste de deux manières. D'une part le caractère effectif de la titularité de certains droits des migrants s'avère particulièrement délicat. C'est

27 En commençant par la Déclaration Universelle des droits de l'homme, le Pacte International relatif aux droits civils et politiques; le Pacte international relatif aux droits économiques, sociaux et culturels; La Convention international sur l'élimination de toutes les formes de discrimination raciale; la Convention sur l'élimination de toutes les formes de discrimination à l'égard des femmes; la Convention contre la torture et autres peines ou traitements cruels, inhumains ou dégradants; la Convention relative aux droits de l'enfant; la Convention pour la répression de la traite des êtres humains et de l'exploitation de la prostitution d'autrui; la Convention contre la criminalité transnationale organisée ainsi que le Protocole relative à la traite des personnes, en particulier des femmes et des enfants et le protocole sur le trafic illicite des migrants par terre, mer et air.

28 Nous trouvons une reconnaissance de la vulnérabilité des migrants dans la Convention internationale sur la protection des droits de tous les travailleurs migrants et des membres de leur famille de 1990 (considérant la situation de vulnérabilité dans laquelle se trouvent fréquemment les travailleurs migrants et les membres de leur famille du fait, entre autres, de leur éloignement de l'Etat d'origine et d'éventuelles difficultés tenant à leur présence dans l'Etat d'emploi,); mais aussi dans les travaux du Haut commissariat des Nations unies pour les droits de l'homme; du Rapporteur des Nations unies pour les droits des migrants; du Rapporteur spécial des Nations unies sur la situation de travailleurs migrants ainsi que dans les conclusions des travaux de la Commission Mondiale sur les migrations internationales, pour n'en citer que quelques uns. 
le cas des droits à une procédure équitable, le droit à l'égalité et à la non discrimination ainsi que l'interdiction des traitements cruels inhumains et dégradants, et l'exercice des droits sociaux en général. Une réflexion autour de la portée théorique et réelle de ces droits est nécessaire pour analyser la possibilité de parvenir à une protection des droits des migrants et à une amélioration de leur situation par le biais de l'invocation de ces droits. Les migrants doivent faire face à toutes sortes de situations discriminatoires. Ces discours s'inspirent de la lecture isolée de certains faits et de leur présentation ou interprétation sélective et peuvent se manifester en actes de violence et de racisme à l'encontre des migrants.

Les migrants sont aussi exposés à la violation de leurs droits dans le cadre du travail. Des horaires excessifs, des rémunérations trop faibles, des difficultés pour porter plainte en cas d'abus et même des cas de travail forcé et de rétention des passeports.

D'autre part, certains groupes de migrants connaissent des situations de vulnérabilité encore plus extrême. Les difficultés décrites deviennent plus marquées s'agissant des femmes et des enfants migrants. Ils sont encore plus exposés aux abus, aux trafics et à la traite, aux travaux forcés et aux discriminations.

S'agissant des migrants en situation irrégulière, leur condition pose aussi de nombreux problèmes. Leur présence sur le territoire des Etats de destination implique une violation des normes internes d'entrée et séjour, violation qui dans certains pays est même considérée comme constituant une infraction pénale ${ }^{29}$. Les migrants en situation irrégulière sont parfois appelés "clandestins ». A cet égard, nombreuses sont les personnalités et organisations, dont le Rapporteur spécial des Nations unies sur les droits de l'homme des migrants; la Commission mondiale sur les migrations internationales; le Haut Commissariat des Nations unies aux droits de l'homme et le Forum mondial sur les migrations et le développement, qui se sont prononcées pour rappeler aux autorités étatiques, mais aussi aux médias et aux personnalités politiques, qu'une personne ne peut «être» clandestine ou irrégulière car il s'agit d'un concept discriminatoire en soi (FMMD, 2010). (Bien différent que de parler de personnes qui se trouvent en situation administrative irrégulière $)^{30}$. Les migrants en situation irrégulière n'ont pas accès aux services sociaux et leurs droits fondamentaux sont parfois méconnus, situation qui constitue une stratégie visant à décourager ce type de migrations, notamment dans le cadre des rétentions administratives. A cet égard certains considèrent que «l'être humain devrait prévaloir sur l'étranger» (Cassese, 1993, p. 236), et que toute personne devrait être traitée avec humanité. Force est de constater que s'agissant des migrants en situation irrégulière, s'est loin d'être le cas.

De manière générale, le problème demeure l'invisibilité des migrants et le manque de véritables instruments pour lutter contre les

\footnotetext{
29 Une étude comparative sur la gestion pénale de l'immigration dans Monclus Maso (2008).

30 Voir, entre autres, Commission mondiale sur les migrations internationales (2005, p 37).
} 
traitements arbitraires et les violations de leurs droits de l'homme, qu'il s'agisse d'une expulsion arbitraire, ou d'un acte d'un employeur sans scrupules qui profite du travail des travailleurs en situation irrégulière, d'un cas de violence sexuelle qui ne peut pas être dénoncé car ses victimes craignent l'expulsion, d'un comportement xénophobe à leur encontre, pour ne mentionner que certaines situations. Les migrants peuvent être la cible de tout type de comportements qui restent dans beaucoup de cas sans protection ni sanction. Pourtant, comme nous l'avons souligné, les pays de destination des migrants sont pour la plupart signataires des principaux traités internationaux de protection des droits de l'homme, mais comme l'a indiqué la Commission mondiale sur les migrations internationales "Certains pays qui ont ratifié les traités fondamentaux des Nations unies relatifs aux droits de l'homme n'appliquent pas les dispositions de ces instruments juridiques, ce qui a pour conséquence l'exploitation, la discrimination et des abus que continuent de connaître de nombreux migrants».

En résumé, le défi de la protection des migrants, au delà, et nous dirons même, malgré la volonté contraire des Etats, est un défi de taille. Nous allons emprunter la notion de Cortes (2005) pour la promotion des droits des femmes migrantes pour dire que ce défi est d'ordre "philosophique, politique et technique ». Philosophique, car il implique la reconnaissance de la vulnérabilité des migrants en général quant à l'accès et la protection de leurs droits fondamentaux; politique pour promouvoir le respect des principaux traités internationaux des droits des l'homme indé- pendamment du statut des migrants ainsi que la ratification de la Convention internationale sur la protection des droits de tous les travailleurs migrants et des membres de leur famille; et finalement technique, pour pouvoir saisir tous les nuances particulières de la dynamique migratoire, et les besoins des migrants qui changent en fonction non seulement des politiques des pays de destination mais aussi des contextes sociaux, politiques et culturels des pays d'origine des migrants.

Devant ce contexte, il s'avère clair que les migrations constituent un phénomène transnational qui a des effets sur trois régimes juridiques différents: en premier lieu, et de la manière la plus évidente, les migrations ont un effet sur le régime juridique interne des Etats de destination. À ce niveau, les migrants sont tenus de respecter les normes du pays de destination dictées dans l'exercice de la souveraineté dudit Etat, lesquelles prévoient les conditions d'entrée et de séjour sur le territoire. En second lieu, les migrations ont des conséquences sur le régime juridique interne des pays d'origine devant faire face à la sortie de leurs nationaux.

Et enfin, le régime international des droits de l'homme qui se met en application lorsque les migrants quittent leur pays d'origine et vont s'installer à l'étranger en tant que titulaires de droits à vocation universelle. La coordination entre ces trois régimes ne s'avère pas simple. Des intérêts divers se mêlent, ainsi que des priorités contradictoires. Toutefois, face à l'arrivée massive de migrants, les Etats de destination ont pris conscience de la nécessité de coopérer et d'intégrer ces trois niveaux. Dans un premier temps, la coopération a eu lieu dans le cadre de relations de voisinage et d'accords 
bilatéraux. Ensuite, le caractère complexe des migrations a amené les pays à rechercher des consensus plus larges pour maîtriser les migrations. C'est ainsi que le concept de «gestion conjointe» est né.

\section{LA GESTION MIGRATOIRE OU GOUVERNANCE MIGRATOIRE INTERNATIONALE}

La gouvernance internationale des migrations, tel que nous l'avons mentionné, est un processus qui promeut la coordination entre différentes autorités et cadres juridiques de régulation migratoire afin d'apporter une réponse conjointe et coordonnée au phénomène et d'encourager la coopération internationale en la matière (HCNUDH, 2012). Or cet exercice de coordination comporte des difficultés. Il s'agit de parvenir à un "équilibre correct» (Pellegrino, 2004, p. 74) entre les différents intérêts autour du phénomène migratoire afin d'en faire un "bien public mondial» (Badie, 2008). Mais il s'agit avant tout d'un exercice dans lequel les Etats doivent céder une partie de leur souveraineté en faveur d'une régulation supra nationale ${ }^{31}$ qui les guidera dans l'exercice de leur prérogative de contrôle migratoire. Les Etats se sont montrés très réticents à accepter des ingérences dans la manière dont ils maîtrisent les migrations. Pour certains cette résistance serait «ironique» car dans la pratique les Etats n'ont jamais possédé un contrôle total sur les migrations (Newland, 2005). A vrai di- re, comme nous l'avons déjà dit, l'idée d'une gouvernance internationale des migrations n'est pas totalement novatrice. Il s'agit de la conséquence d'une réalité incontestable: les migrations sont un phénomène transnational et en tant que tel doivent faire l'objet d'un traitement transnational. Comme le dit l'oim «les migrations ayant toujours existé et constituant un mouvement naturel des êtres humains (plus naturel d'ailleurs que les frontières qu'ils ont à franchir), il n'est pas surprenant que les Etats s'associent pour organiser ces mouvements» (oIm, 2002, p. 12).

Cette gouvernance internationale des migrations s'opère sous diverses formes. D'après ce qui a été établi par la Commission mondiale sur la migration internationale, elle comprend la construction de politiques publiques et des normes internes des Etats en la matière, les discussions bilatérales et multilatérales entre Etats sur le sujet; et enfin les forums multilatéraux, processus consultatifs et activités des organisations internationales dédiés à la question Commission Mondiale sur les migrations internationales, 2005, p. 65).

Compte tenu du contexte complexe qui vient d'être présenté, des multiples intérêts qui sont en jeu dans la gouvernance des migrations et des besoins des personnes migrantes, nous pouvons nous demander: Quel serait le scenario optimal pour maîtriser les migrations tout en protégeant les droits des migrants?

La première configuration possible consiste en une gouvernance mondiale des mi-

31 Pour le Rapporteur spécial des Nations unies sur les droits des migrants il ne s'agit pas de céder une partie de la souveraineté mais de la regagner (Assemblée générale 2013). 
grations, scénario visant à générer un consensus mondial sur la gouvernance des migrations à travers la promotion de réflexions, d'études et de programmes conjoints. Il pourrait constituer le meilleur espace de gouvernance des migrations tout en protégeant les droits des migrants du fait de ses échanges de niveau mondial qui invitent à une réflexion conjointe entre points de vue divers.

Les efforts pour aboutir à ce type de gouvernance ont eu lieu à partir des années quatrevingt-dix, dans le cadre ou avec le soutien de l'onu et avec une nuance particulière: il s'agit d'espaces de discussion qui cherchent à mâ̂triser les migrations afin d'en faire un outil du développement. La Conférence internationale sur la population de 1994 (au Caire) a marqué un tournant décisif dans le débat sur les migrations et le développement et de ce fait dans la création d'espaces de discussion migratoire visant à générer un consensus à l'échelle mondiale. Le programme d'action a dédié un chapitre aux migrations internationales et a invité les Etats à «accorder plus d'importance à la question dans les politiques relatives à la population et au développement» ${ }^{32}$ mais aussi à coopérer dans la gestion migratoire. Dès lors, le sujet a été inclus dans toutes sorte d'agendas de discussion et débats ${ }^{33}$.

Le second scenario possible pour parvenir à une gouvernance optimale des migra- tions, respectueuse des droits des migrants, serait celui de la gouvernance régionale des migrations.

En effet, le sujet de la gouvernance des migrations a également été abordé dans le cadre des organisations régionales de coopération et d'intégration. Ces dernières regroupent des pays voisins ou proches, qui partagent des cultures similaires et se sont mis d'accord pour gérer de manière coordonnée certaines questions, comme celle de la gestion migratoire. Ainsi la gestion régionale des migrations vise à adopter des mesures communes et des principes directeurs pour régler la circulation des ressortissants des Etats membres ainsi que celle des étrangers en provenance d'Etats tiers. Ces structures peuvent également définir une position commune pour répondre à la question de la protection des ressortissants des Etats membres à l'étranger. Dans certains cas, elles ont même établi des autorités communes pour la surveillance des frontières. Par exemple, dans la région andine et l'Amérique latine, tout un ensemble d'organisations politiques de coopération et d'intégration coexistent. Certaines ont un caractère plutôt politique, telles que l'Organisation des Etats Américains (oEA); l'Union des Nations sud américaines (UNAsUR); le Groupe de Rio (récemment transformé en CELAC). D’autres ont une ambition économique qui vise l'intégration de leurs Etats

\footnotetext{
32 Rapport de la Conférence Internationale su la population et le développement Le Caire 5- 13 Novembre 1994. A/CONF.171/13/Rev.1.

33 Tels que les sommets mondiaux pour le développement social de 1995, la quatrième Conférence mondiale sur les femmes de 1995 et ses quatre vingt-quatrième et vingt-cinquième sessions extraordinaires ainsi que le Sommet mondial de 2005 .
} 
membres, comme la Communauté andine des nations (CAN); le Mercosur et l'Alliance du Pacifique.

Ces structures ont abordé le sujet migratoire dans plusieurs buts: d'une part, les organisations à vocation économique ont compris que la création d'un marché ouvert passait par la maîtrise de la liberté de circulation des personnes à l'intérieur des frontières des Etats membres; à cet égard, des mesures ont été définies pour garantir et faciliter la circulation des citoyens des Etats membres et associés à l'intérieur de leurs frontières, à travers la reconnaissance des diplômes, la dispense de visas, mais aussi la protection des droits du travail et l'application de certaines garanties spéciales pour les travailleurs en termes d'équivalences des périodes de cotisation à la retraite. D'autre part, les structures à caractère politique ont émis des déclarations et des propositions dans lesquelles elles s'engagent à coopérer en matière migratoire, afin notamment d'encourager la coopération, l'échange de données et la coordination des dispositions nationales.

Enfin, le sous-continent dispose de deux processus consultatifs régionaux en matière de migrations: le processus consultatif de Puebla et la Conférence sud-américaine des migrations, cadres de discussion à caractère non contraignant qui ont sensiblement contribué à la compréhension du phénomène et à l'adop- tion d'une position conjointe, notamment face aux migrations extracontinentales.

Par ailleurs, la gouvernance des migrations dans le cadre des relations interrégionales et bilatérales apparaît comme une troisième hypothèse à explorer. En effet, le contexte migratoire, les liens historiques et les rapprochements commerciaux entre l'Amérique latine et l'Union européenne ont donné lieu à la formation d'espaces de discussion qui se sont développés à trois niveaux: en premier lieu, au niveau du sous-continent dans son ensemble, (dans le cadre des dialogues Union européenne-Amérique latine et Caraïbes); en deuxième lieu, dans le cadre des relations avec des organisations du sous-continent (dans les cadre des accords de coopération entre l'Union européenne et la Communauté andine par exemple) et enfin dans le cadre des relations bilatérales entre l'Union et certains pays du sous-continent (tels que la Colombie et le Pérou qui ont conclu des accords de libre échange avec l'UE). Ces échanges ont abouti à la conclusion d'accords de coopération dans lesquelles la question migratoire a été abordée, dans une perspective de contrôle migratoire ${ }^{34}$.

Le quatrième scenario possible pour parvenir à une gouvernance optimale des migrations, serait celui de la gestion migratoire respectueuse des droits des migrants opérée par les Etats d'origine. Traditionnellement, les études

\footnotetext{
34 A cet égard, l'Union européenne dans le programme de La Haye de 2004 a inclus, parmi les dix sujets prioritaires pour les cinq prochains années, la définition d'une «approche équilibrée concernant la migration» où il était mentionné qu' " une politique commune en matière d'immigration ne peut se borner aux mesures d'admission et de retour: une gestion efficace des flux migratoires doit comporter un sérieux investissement dans les relations avec les pays tiers, d'origine comme de transit, notamment par l'intermédiaire de l'assistance et de la coopération, dans l'intérêt mutuel des pays tiers et de l'Union» (Commission des communautés européennes, 2005).
} 
sur les migrations ont tenu compte de l'impact des migrations sur les pays de destination des migrants car c'est dans ces pays des transformations visibles s'opèrent du fait de l'arrivée massive de personnes étrangères. L'impact des migrations dans les pays d'origine ne s'est fait sentir que par la suite, lorsque l'arrivée massive de flux d'argent en provenance des migrants vers les membres de leurs familles restées au pays a commencé a attirer l'attention des banques et des intermédiaires financiers, puis des autorités publiques et des chercheurs.

C'est notamment le cas de la Colombie, où les premières études sur l'impact du phénomène ont été menées dans le cadre d'un projet de recherche sur le profil des destinataires du transfert de fonds (Mejía, 2011, p. 105). Or la diaspora colombienne, tout comme celle de l'Equateur, représente près de $10 \%$ de la population. Le nombre estimé de colombiens à l'étranger est d'environ quatre millions ${ }^{35}$.

Pour finir, une cinquième hypothèse pour gouverner les migrations tout en protégeant les droits des migrants serait de justement donner pleine application aux droits des migrants consacrés dans les principaux traités internationaux des droits de l'homme.

L'étude des droits des migrants implique de sortir de la logique classique gestion/ contrôle dont s'inspirent les principaux scénarios de gouvernance migratoire. Il s'agirait en effet d'appréhender les migrants en tant que personnes, pour tenir compte de leurs besoins ponctuels et des difficultés qu'ils rencontrent à l'heure d'exercer certains droits et de s'adapter aux sociétés de destination. Une telle conception permettrait de doter la gouvernance migratoire d'une dimension humaine.

La protection des droits des migrants a intéressé dans un premier temps l'Assemblée générale des Nations unies, qui, dans le cadre de sa troisième Commission a encouragé l'élaboration d'études sur les droits des non ressortissants ${ }^{36}$, ainsi que des rapports sur les droits des migrants. Elle a également convoqué la communauté internationale afin d'élaborer une Convention internationale sur la protection des droits de tous les travailleurs migrants et des membres de leur famille. Cet instrument date de 1990, mais son application s'est avérée compliquée du fait de sa faible ratification par les principaux pays de destination des migrants.

De la même manière, dans le cadre du Système interaméricain de protection des droits de l'homme, la Commission tout comme la Cour ont contribué à l'analyse, la compréhension et la dénonciation des besoins particuliers des

\footnotetext{
35 La population colombienne est de 42.888.594 d'après les chiffres officiels du Département national des statistiques. Le nombre de colombiens à l'étranger est tiré des estimations faites par ce même Département à partir des donnés récoltées lors du recensement national de 2008, des chiffres des consulats et les chiffres des postes d'entrée et de sortie du pays.

36 En1980, Elles Baroness, experte de la Sous-commission pour la promotion et protection des droits de l'homme, a rendu un rapport intitulé Dispositions internationales pour la protection des droits de l'homme des non-ressortissants, E/ CN.4/Sub.2/392/Rev.1 (1980). De la même manière, David Weissbrodt a présenté un rapport dans lequel il a noté des différences dans les cadres normatifs applicables aux non-ressortissants. Rapport final du Rapporteur spécial, David Weissbrodt, E/CN.4/Sub.2/2003/23.
} 
migrants dans les pays membres du système, grâce aux travaux du Rapporteur spécial sur la protection des droits des migrants (appartenant à la Commission) mais aussi des avis consultatifs et des arrêtés de la Cour.

Cette analyse succincte, des causes et des conséquences des migrations, ainsi que des différents espaces pour la gouvernance des migrations nous permet de tirer quelques conclusions. Nous sommes devant un phénomène qui touche à plusieurs aspects sensibles pour les Etats et la Communauté international dans son ensemble tels que la politique, l'économie, la sécurité et la protection des droits de l'homme. Malgré l'importance du sujet et les multiples espaces dans lesquels il a été porté, discuté et analyse, nous ne pouvons dire, au jour d'au jour d'huit, qu'il existe un cadre idéal pour la gouvernance des migrations qui soit respectueux des droits des migrants au même temps qu'il réponde aux intérêts étatique et soit le produit des concertations interétatiques. Bien au contraire, les réponses au phénomène continuent à être disperses et même improvisées. Le défi de la gouvernance migratoire reste de taille, nous considérons cependant, que la maîtrise des migrations passe par la compréhension du phénomène, de ces causes et conséquences ainsi que par l'effort conjoint de tous les espaces de discussion, débat et gestion migratoire.

\section{BIBLIOGRAPHIE}

Ardila, G. (ed) (2006). Colombia: migraciones, transnacionalismo y desplazamiento. Bogotá: Facultad de ciencias Humanas UN. Colecciones CES.
Badie, B. et al. (2008). Pour un autre regard sur les migrations. Construire la gouvernance mondiale. Paris: La découvert.

BID (2010). Las remesas a América Latina y el Caribe durante el 2009. Los efectos de la crisis financiera global. Banque Interaméricaine de Développement, Fonds multilatéral d'investissements. Recuperado de http://idbdocs.iadb.org/wsdocs/getdocument. aspx?docnum $=35101520$

Ayuso, A. et Pinyo, G. (2010). Inmigración Latinoamericana en España, el estado de la investigación. СІDов.

Cassese, A. (1993). Les droits de l'homme dans le monde contemporain. Barcelona: Ariel.

CIISE (2001). La responsabilité de protéger: Rapport de la Commission internationale de l'intervention et de la souveraineté des Etats. Canadá: Centre de recherches pour le développement international.

Commission Mondiale sur les migrations internationales (2005). Les migrations dans un monde interconnecté. Nouvelles perspectives d'action. Rapport de la Commission mondiale sur les migrations internationales.

Cervantes Gabarrón, J. (2008). Los derechos del inmigrante en la biblia. Pastoral de migraciones de la parroquia. Pastoral social.

Cortes, P. (2005). Mujeres migrantes en América Latina y el Caribe: Derechos humanos, mitos y duras realidades. Santiago de Chile: Comisión económica para América Latina y el Caribe (CEPAL), Pulicación de las Naciones Unidas.

De Soigne, J. (1873). Traité du Droit des Etrangers en Belgique.

FMMD (2008). Rapport de la première réunion du Forum mondial sur la migration et le développement. Bruylant Bruxelles.

FMMD (2010). Partenariats pour la migration et le développement humain: prospérité partagée - responsabilité partagée. Puerto Vallarta: Quatrième réunion du 
Forum mondial sur les migrations et le développement.

Gourévitch, J. P. (2009). Limmigration ça coûte ou ça rapporte? France: Larousse.

Gincel, A. (2007). Santuario un village colombien à Paris. Dans Migrations latino-américaines. Homme et migrations.

Guarnizo, L. E. (2006). Sobre las representaciones de las migraciones en ciencias sociales. Dans Ardila, G. (ed). Colombia: migraciones, transnacionalismo y desplazamiento. Bogotá: Facultad de ciencias Humanas un.

HCNUDH (2012). Migrations and human rights. Improving human rights based governance of international migration. Dans Betts, A. (ed.). Global Migration Governance. Oxford: Oxford University Press.

Kymlicka, W, (2002). ciudadanía multicultural. Paidos. Buenos Aires. 2002

Madiedo, M. M. (1863). Droit des gens. Boston.

Martínez Pizarro, J. (ed.) (2008). América Latina y el Caribe: migración internacional, derechos humanos y desarrollo. Santiago de Chile: Comisión económica para América Latina y el Caribe (CEPAL), Publicación de las Naciones Unidas.

Martínez Pizarro, J (2010). Cambios y desafios en la migración internacional. Dans Ayuso, A. et Pinyo, G. Inmigración Latinoamericana en España, el estado de la investigación. СІDов.

Martínez Pizarro, J. (2003). El mapa migratorio de América latina y el Caribe, las mujeres y el género. Santiago de Chile: CELAde, Fondo de Población de las Naciones Unidas.

Masson, B. (2010). Mineurs isolés Etrangers: Le sens d'une appellation. Migrations Société, 22 (129-130).

Mattoo, Aaditya, Neagu et Özden (2005). Brain waist Educated immigrants in the us labor market. World Bank Policy Research Working Paper Series 3581, Washington, DC.: World Bank.
Mejía, W. (2011). Politica, normatividad, acciones y adecuación institucional del Estado colombiano con relación a las migraciones laborales, a través de su historia. OIT MIGRANADINA.

Monclus Maso, C. (2008). La gestión penal de la inmigración: El recurso al sistema penal para el control de los flujos migratorios. Buenos Aires.

Newland, K. (2005). The gouvernance of International migration: mechanisms, processes and institutions. Paper prepared for the Policy Analysis and Research Programme of the Global Commission on International Migration. CMMI, Migration Policy Institute.

Office fédéral des migrations odM, oIm (2004). Agenda international pour la gestion des migrations: perceptions communes et pratiques efficaces sinscrivant dans une approche d'ensemble planifiée et équilibrée de la gestion des migrations. Berne.

OIM (2007). Glossaire de la migration. Genève Suisse: Droit international de la migration.

OIM (2010).Les migrants Latino-Américains en Europe. 11 ème forum Biarritz.

OIM (2002). International Dialogue on Migration N.2. Compendium of intergouvernmental organisations active in the field of migration. Genève: PPMR, OIM. Pellegrino, A. (2004). Migrations from Latin America to Europe: Trends and policy challenges. Uruguay: OIM.

Rannveig Agunias, D. (comp.) (2009). Closing the Distance: How Governments Strengthen Ties with Their Diasporas, Migration Policy Institute (MPI). Washington, D.C.

Saïd, E. (2005). Réflexions sur l'exile et d'autres essais. Barcelona.

Sassen, S. (2001). ¿Perdiendo el control? La soberania en la era de la globalización. Barcelona: Ediciones Bellaterra. 
Villegas Pulido, InICIAL. (1919). Los extranjeros en Venezuela: Introduccion historia de movimientos migratorios, Su no admisión-Su expulsión. Caracas: Lit y TIp del comercio.

\section{Documents des Nations Unies}

Assemblée Générale des Nations Unies (2013). Formes contemporaines de racisme, de discrimination raciale, de xénophobie et de l'intolérance qui y est associée. Rapport du Rapporteur spécial. A/HRc/23/56 2. Assemblée Générale (2002). Droits de l'homme des migrants. Rapport présenté par la Rapporteuse spéciale sur les droits de l'homme des migrants. A 57/292 du 9 août 2002.

Assemblée générale (2002). Résolution 56/203. Assemblée générale (2006). Résolution 60/871.
Assemblée générale des Nations unies (2005). World Summit Outcome, Résolution 60/1.

Commission des communautés européennes (2005). Programme de La Haye: dix priorités pour les cinq prochaines années Un partenariat pour le renouveau européen dans le domaine de la liberté, de la sécurité et de la justice,. Communication de la Commission au Conseil et au parlement européen. Recuperado de http://eur-lex.europa.eu/LexUriServ/ LexUriServ.do?uri=CELEX:52005PC0184:FR:NOT Commission des droits de l'homme (1999). Rapport $d u$ Groupe de travail intergouvernemental d'experts sur les droits de l'homme des migrants. Résolution $1999 / 80$.

PNUD (2009). Rapport mondial sur le développement humain 2009: lever les barrières: mobilité et développement humains. ONU. 